\title{
Comparing the Biomechanical Stability of Cortical Screw Trajectory Versus Standard Pedicle Screw Trajectory for Short- and Long-Segment Posterior Fixation in 3-Column Thoracic Spinal Injury
}

\author{
AMEY R. SAVARDEKAR, MD, MCH, NESTOR G. RODRIGUEZ-MARTINEZ, MD, ANNA G.U.S. \\ NEWCOMB, MS, PHILLIP M. REYES, BSE, HECTOR SORIANO-BARON, MD, STEVE W. CHANG, MD, \\ BRIAN P. KELLY, PHD, NEIL R. CRAWFORD, PHD \\ Department of Neurosurgery, Barrow Neurological Institute, St. Joseph's Hospital and Medical Center, Phoenix, Arizona
}

\begin{abstract}
Background: Information on the performance of posterior fixation with cortical screw (CS) versus pedicle screw (PS) trajectories for stabilizing thoracolumbar burst fractures is limited. Therefore, we sought to analyze stability with CS versus PS in short- and long-segment fixations using a 3-column spinal injury model.

Methods: Nondestructive flexibility tests: (1) intact, (2) intact + short fixation, (3) intact + long fixation, (4) after burst fracture, (5) short fixation + burst fracture, and (6) long fixation + burst fracture using thoracic spine segments (7 CS, 7 PS).

Results: With CS, the range of motion (ROM) was significantly greater with short-segment than with long-segment fixation in all directions, with and without burst fracture $(P \leq .008)$. With PS and burst fracture, ROM was significantly greater with short fixation during lateral bending and axial rotation $(P<.006)$, but not during flexion-extension $(P=.10)$. Groups with CS versus PS were not significantly different after burst fracture during flexion-extension and axial rotation, with short $(P \geq .58)$ or long fixation $(P \geq .17)$. During lateral bending, ROM was significantly greater with CS versus PS, without burst fracture (long fixation, $P=.02$ ) and with burst fracture (short and long fixation, $P \leq .001$ ).

Conclusions: CS trajectory is a valid alternative to PS trajectory for thoracic spine fixation in 3-column spinal injuries, and long-segment fixation is superior to short-segment fixation with either.
\end{abstract}

Biomechanics

Keywords: burst fracture, construct length, cortical screw, flexibility, pedicle screw, thoracolumbar biomechanics

\section{INTRODUCTION}

Most thoracolumbar injuries can be effectively treated with conservative management ${ }^{1,2}$; however, some patients with 3-column injuries require surgery. The standard technique for unstable thoracolumbar fractures is pedicle screw (PS) and rod fixation. ${ }^{3-6}$ Unstable thoracolumbar fractures have traditionally necessitated long-segment fixation (2 levels above, 2 levels below) with screw and rod constructs for rigidity. ${ }^{7,8}$ Recent studies have shown the efficacy of short-segment fixation (1 level above, 1 level below) posterior constructs compared with long-segment fixation. ${ }^{8,9}$

As an alternative to PSs for posterior fixation, cortical screws (CSs) are becoming more common in lumbar spinal fusion surgeries. ${ }^{3,10-13}$ Compared with PSs, CSs require a more sagittally and cranially oriented trajectory into the pedicle and are anchored in the pars interarticularis (Figure 1). ${ }^{3,14}$ A previous biomechanical study showed that, by immediate postoperative stability alone, CSs are a valid alternative to PSs for lumbar spinal fixation. ${ }^{3}$ Although the pars in the thoracic spine includes less cortical bone than in the lumbar spine, CSs can also be used for thoracic spinal fixation. In a biomechanical study including morphometric and insertion torque measurements in thoracic spine segments, CSs were shown to be feasible and to have favorable screw fixation stability compared with a traditional PS technique, warranting additional biomechanical analysis. $^{12}$

This study was performed to test the hypothesis that the stability conferred by CS-and-rod fixation in the thoracic spine is equivalent to that of PS-and- 

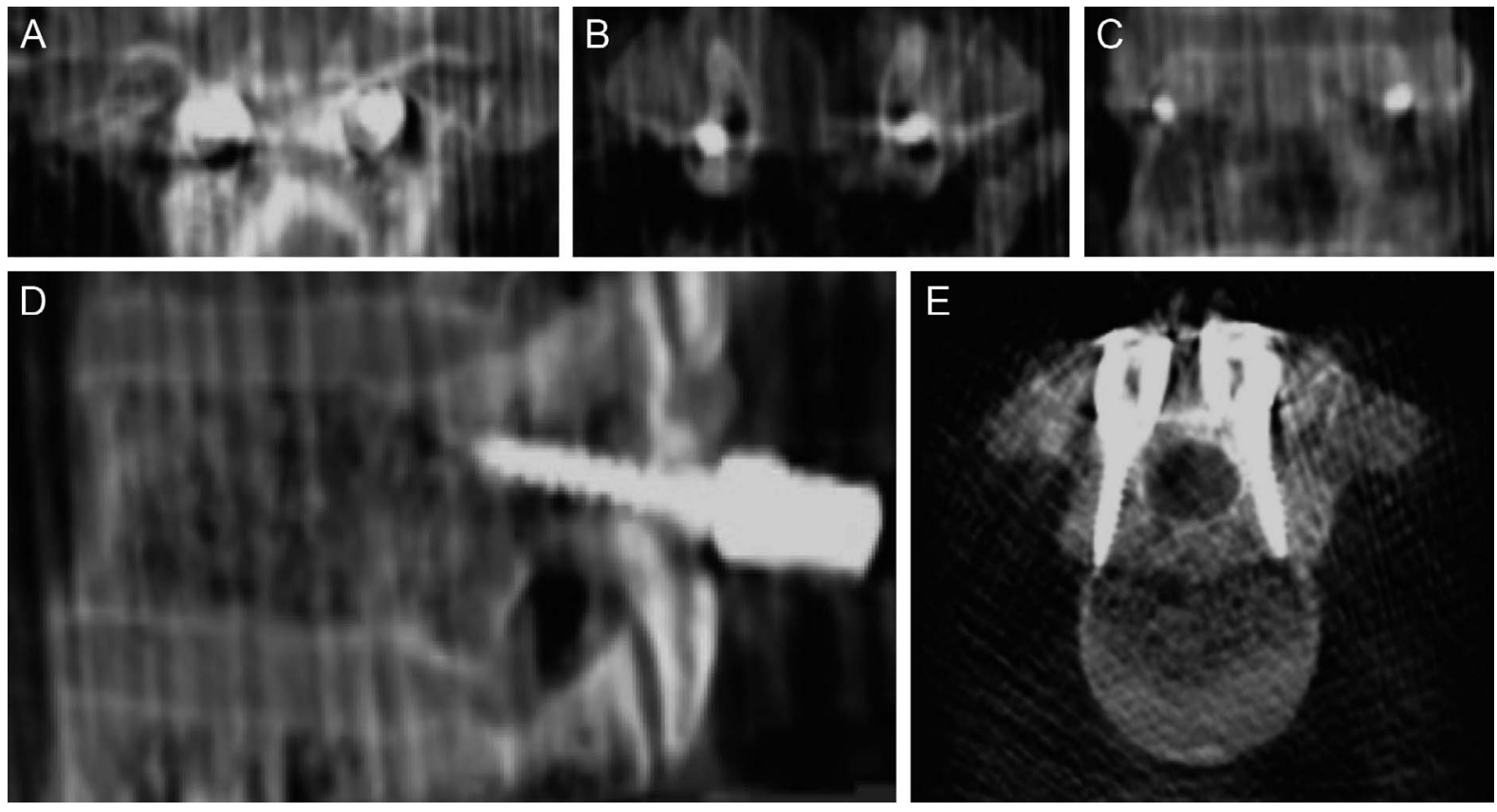

Figure 1. Coronal (A, plane of entry; B, inside pedicles; and C, plane at screw tip), (D) lateral, and (E) axial views of the cortical screw trajectory at T1. Used with permission from Barrow Neurological Institute, Phoenix, Arizona.

rod fixation in a 3-column spinal injury model. The hypothesis was tested in short-fixation and longfixation constructs. Stability was defined as reduced mobility during loading, in terms of the angular range of motion (ROM) during flexibility tests and vertical displacement during axial compressive tests.

\section{MATERIALS AND METHODS}

\section{Specimen Preparation}

Fourteen human cadaveric thoracic spine segments (2 T5-T11 and 12 T6-T12) were used. All specimens had ribs transected approximately $4 \mathrm{~cm}$ from the costovertebral joints and were of similar length. Fresh frozen specimens were thawed in normal saline at $30^{\circ} \mathrm{C}$ and cleaned of muscle tissue without damaging ligaments, discs, or joint capsules. Specimens were then examined for obvious flaws and acceptable bone mineral density.

The heads of household screws were inserted in the distally exposed endplates, and posterior structures of the proximal and distal vertebrae were embedded in metal fixtures using fast-curing resin (Smooth-Cast 300Q, Smooth-On, Inc., Easton, Pennsylvania) and attached to the base and loading pulley of the testing apparatus.

The specimens were separated into 2 groups matched by age (PS: $50.6 \pm 11.7$ years versus CS: $50.4 \pm 9.4$ years); sex (PS: 5 men and 2 women versus CS: 6 men and 1 woman); and bone mineral density (PS: $0.724 \pm 0.143 \mathrm{~g} / \mathrm{cm}^{2}$ versus CS: $\left.0.755 \pm 0.207 \mathrm{~g} / \mathrm{cm}^{2}\right)$. Specimens were tested in 6 conditions: (1) intact; (2) intact + short fixation; (3) intact + long fixation; (4) after burst fracture; (5) short fixation + burst fracture; and (6) long fixation + burst fracture. The order of testing of shortsegment versus long-segment fixation before and after burst fracture was systematically varied among specimens to prevent bias. That is, steps 2 and 3 were inverted with steps 5 and 6 in half the specimens. Burst fracture was simulated by creating channels in bone with a thin fluted drill bit (The Anspach Effort, Inc., West Palm Beach, Florida) that involved the superior one-third of the vertebral body, including the posterior cortex, and proceeded laterally to the pedicles at the index (fracture) level (T8 $[\mathrm{n}=2]$ or T9 [n=12]). The anterior longitudinal ligament was cut anteriorly, and the supraspinous and interspinous ligaments were cut posteriorly, rostrally, and caudally to the index site. The posterior longitudinal ligament was kept intact. The resulting injury model, involving both anterior and middle columns, was severely unstable and caused segmental kyphosis (Figure 2). ${ }^{9}$

\section{Surgical Procedure}

In the PS group, stabilization consisted of shortsegment (1 level above and 1 level below the index 


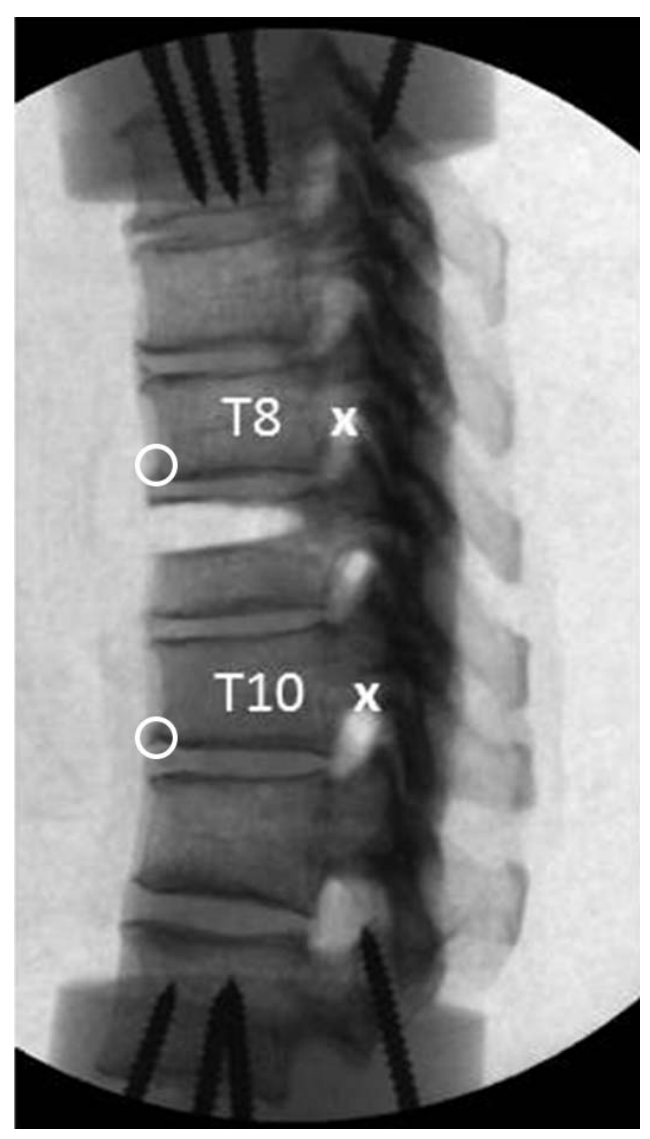

Figure 2. Lateral view radiograph shows the modeled burst fracture at T9. Anterior $(\mathrm{O})$ and posterior $(\mathrm{X})$ anatomic landmarks were used for tracking during motion analysis and for calculations of vertical displacement during compressive loading. Bone at the superior one-third of the vertebral body, including the posterior cortex and proceeding laterally to the pedicles, was removed using a high-speed drill. The posterior longitudinal ligament was kept intact. Used with permission from Barrow Neurological Institute, Phoenix, Arizona.

level, which was T8 in the 2 T5-T11 specimens and T9 in the 12 T6-T12 specimens) and long-segment fixations ( 2 levels above and 2 levels below the index level, which was T8 in 2 specimens and T9 in 12 specimens) using 4.5-mm screws, rods, and no crosslink. The index level was not instrumented and hence was not included in the construct. Stabilization in the CS group consisted of short and long fixations with $4.0-\mathrm{mm}$ screws, rods, and no crosslink. Screws were placed under O-arm guidance to assess trajectory. Montages were tightened after adequate postural correction, with the restoration of thoracic curvature as observed in the intact specimen.

\section{Testing Protocols}

Specimens were subjected to pure moment flexibility tests and axial compression tests (MTS Systems Corp., Eden Prairie, Minnesota) (Figure 3). ${ }^{15}$ With pure moment flexibility tests, the load is
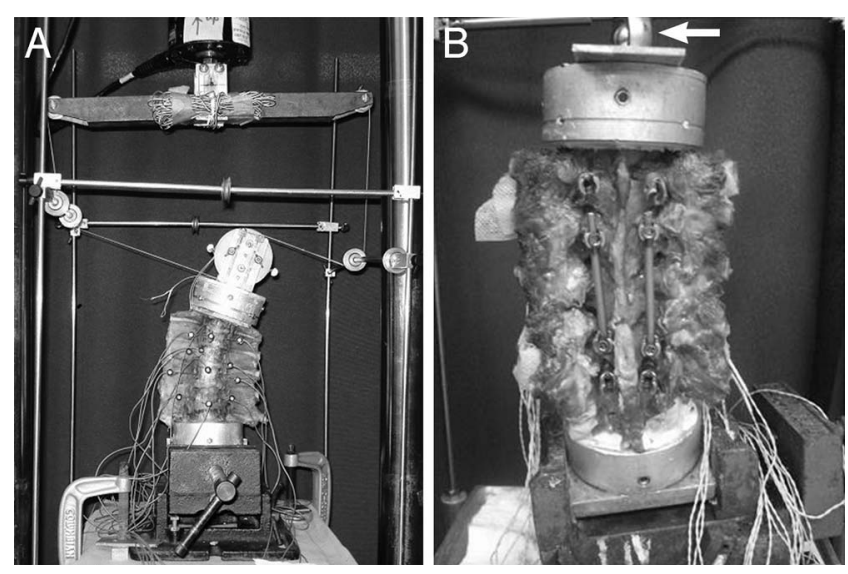

Figure 3. Test configurations for pure moment (A) angular flexibility loading and $(B)$ compression loading performed in a standard servo-hydraulic test frame (MTS Systems Corp., Eden Prairie, Minnesota). During flexibility tests, adjustable pulleys were used, enabling 2 equal and opposite forces separated by a small distance (pure moment) to be applied via a loop of string when the piston advanced upward. Optical markers for tracking specimen motion are also visible. Shown for $(\mathrm{A})$ left lateral bending; reconfiguring the string and pulleys allows for flexion, extension, or axial rotation. The angle vise securing (B) the construct was repositioned on the base of the test frame in the transverse plane until minimal intervertebral rotations were observed during application of the compressive load via the piston (arrow). Shown for short-segment fixation with cortical screws and rods (distal and proximal screws in place but not attached to rods, posterior view). Used with permission from Barrow Neurological Institute, Phoenix, Arizona

distributed evenly to each motion segment, regardless of the distance from the loading point. ${ }^{16}$ Loads (6.0 $\mathrm{Nm}$ maximum) were applied about the anatomical axes to induce 3 types of motion: flexionextension, lateral bending, and axial rotation. Loads were applied quasi-statically in $1.5-\mathrm{Nm}$ increments (held 45 seconds), following 3 preconditioning cycles at maximum load (60 seconds) to allow for creep.

Nonconstraining axial compression tests were performed after flexibility bending tests, with vertical compression loading applied to each construct using the piston on the servo-hydraulic apparatus (Figure 3B). Axial compression was slowly applied (approximately $1 \mathrm{~N} / \mathrm{sec}$ ) to a maximum of $300 \mathrm{~N}$ and held for 45 seconds before recording final intervertebral positions to allow for creep. Axial displacement was determined as the change in vertical height across the index level (T8T10 [n=12] or T7-T9 $[\mathrm{n}=2])$, anteriorly and posteriorly ("O" and "X", respectively, in Figure 2), during load application, as determined from tracking these digitized points during motion analysis.

Intervertebral rotations and translations in response to applied loads were tracked using the Optotrak 3020 system (Northern Digital, Inc., Waterloo, Ontario, Canada), as previously de- 
Table 1. Intact ROM (degrees) across T8-T10.

\begin{tabular}{lrrc}
\hline & CS group & PS group & $P$ value \\
\hline Flexion-extension & $5.0 \pm 0.9$ & $6.8 \pm 1.6$ & .03 \\
Axial rotation & $11.4 \pm 1.0$ & $14.2 \pm 3.6$ & .07 \\
Lateral bending & $9.2 \pm 2.3$ & $9.7 \pm 2.5$ & .67 \\
\hline
\end{tabular}

Abbreviations: CS, cortical screw; PS, pedicle screw; ROM, range of motion.

scribed. ${ }^{17}$ Spinal angles in anatomical planes were calculated using a technique that quantifies the angular coupling of joints. ${ }^{18}$

\section{Statistical Methods}

During motion in all planes, the angular ROM was determined at each level from angle versus moment data. ${ }^{19}$ In each group (CS and PS), data representing motion across 1 level above and 1 level below the index level (T7-T9 or T8-T10) were analyzed using 1-way repeated-measures analysis of variance and the Holm-Šidák test to determine whether mean outcome measures were significantly different among test conditions. The nonpaired 2tailed Student $t$ test was used to compare corresponding mean outcomes in the PS and CS groups. To mitigate effects of variability among specimens before statistical analysis, we also analyzed normalized ROM data for each specimen by dividing the outcome in the surgically altered condition by the corresponding outcome in the normal intact condition, thereby providing a dimensionless ratio to intact. Significance was defined as $P<.05$. Data are presented as mean $\pm \mathrm{SD}$ values.

\section{RESULTS}

The intact ROM was significantly greater in the PS versus the CS group during flexion-extension (Table $1, P=.03$ ), but there were no differences between groups (intact states) during axial rotation or lateral bending $(P>.05)$. The 3-column injury simulating a burst fracture created severe instability, especially during flexion-extension, with 4 to 6 times greater ROM than normal (Figure 4). Destabilization resulted in equivalent ROM (PS versus CS groups) during flexion-extension (Figure 4A, $P=.76$ ) and lateral bending (Figure $4 \mathrm{~B}, P=.70$ ), but greater $\mathrm{ROM}$ in the PS group during axial rotation (Figure $4 \mathrm{C}, P=.04$ ).

There were no statistically significant differences between CS and PS during any condition in flexionextension (Figure $4 \mathrm{~A}, P \geq .40$ ) or axial rotation (Figure $4 \mathrm{C}, P \geq .17$ ). During lateral bending, the ROM with CS was significantly greater than with PS in both long fixation with and without burst fracture (Figure 4B, $P<.02$ ) and short fixation with burst fracture $(P=.001)$.

For the PS group, ROM across T8-T10 (or T7T9) without burst fracture was significantly greater with short fixation than with long fixation during flexion-extension and lateral bending (Figure 4, $P<.001)$. With burst fracture, short fixation with PS was significantly less stable (larger ROM) than long fixation with PS during lateral bending and axial rotation (Figure $4, P \leq .006$ ). The difference between short and long fixation with PS and burst fracture was not significant during flexion-extension (Figure 4, $P=.10$ ).

For the CS group, ROM with short fixation was significantly greater than with long fixation in all directions, both with and without burst fracture (Figure $4, P \leq .008)$.

For conditions before burst fracture, mean changes in vertical height across T8-T10 (or T7T9) during compressive loading were similar anteriorly and posteriorly for the CS and PS
A

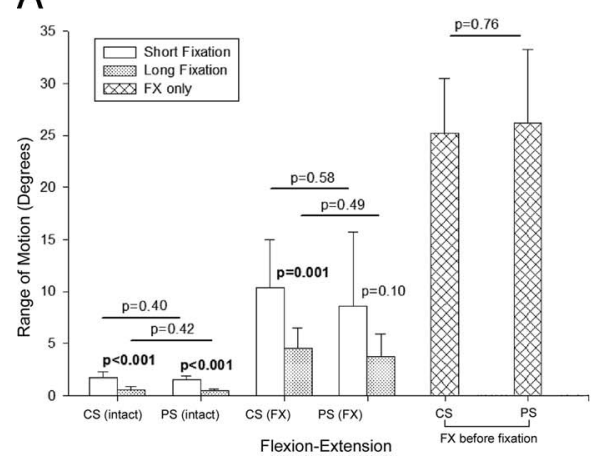

B

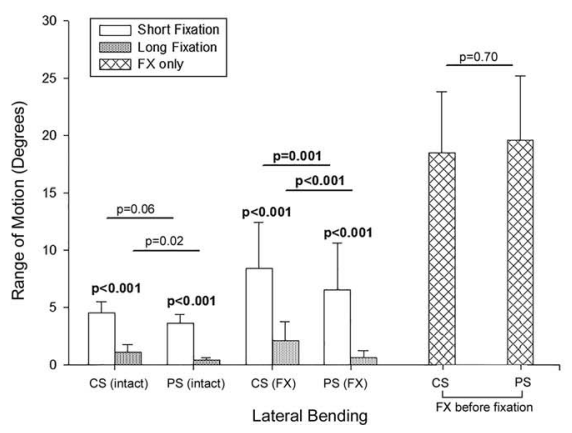

C

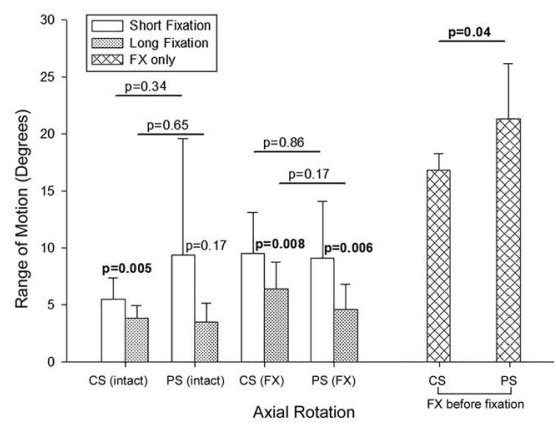

Figure 4. Mean range of motion across T8-T10 (or T7-T9) for all conditions (intact vertebrae, FX with short-segment and long-segment fixation, and FX before fixation). (A) Flexion-extension, (B) lateral bending, and (C) axial rotation. $P$ values are based on statistical analysis of raw values of range of motion. Error bars indicate SD. Abbreviations: CS, cortical screw; FX, burst fracture; PS, pedicle screw. Used with permission from Barrow Neurological Institute, Phoenix, Arizona. 


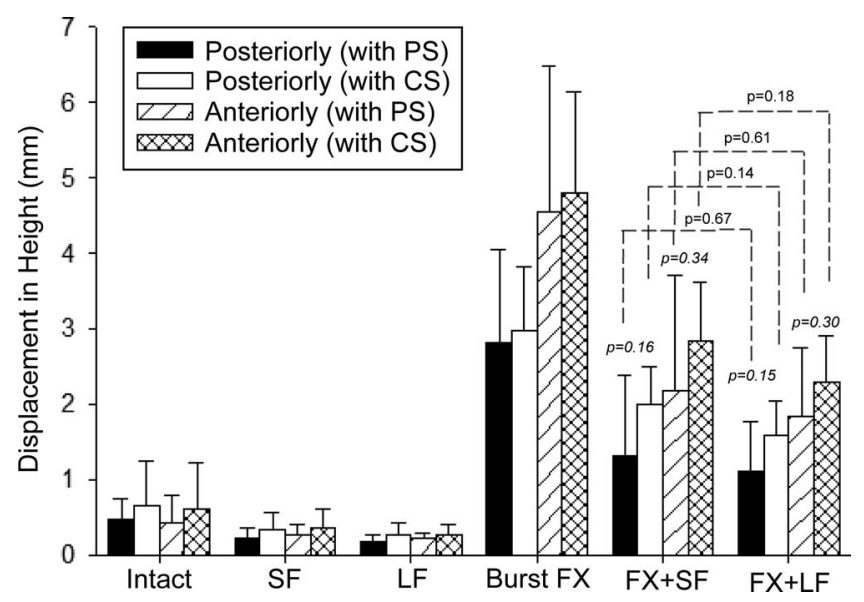

Figure 5. Mean changes in rostrocaudal height across T8-T10 (or T7-T9), anteriorly and posteriorly, during $300-\mathrm{N}$ axial compression for all conditions. $P$ values shown in italics are for differences between PS and CS with fracture plus fixation $(P \geq .15)$. $P$ values shown atop dashed lines are for differences between short and long fixations within CS and PS groups $(P>.14)$. Error bars indicate SD; CS, cortical screw; FX, burst fracture; LF, long fixation; PS, pedicle screw; SF, short fixation. Used with permission from Barrow Neurological Institute, Phoenix, Arizona.

groups and short and long fixations (Figure 5), indicating minimal intervertebral rotations. With a simulated burst fracture, the posterior vertical height changes were $606 \% \pm 263 \%$ of intact in the PS group and $450 \% \pm 126 \%$ of intact in the CS group (Figure 5). With burst fracture, the vertical height changes were somewhat greater anteriorly than posteriorly in CS and PS groups and with short and long fixations (Figure 5), indicating flexural rotations. Overall, mean changes in vertical height during compression tests (anterior and posterior) were slightly greater in CS than PS groups for all conditions. However, these differences were not statistically significant (Figure 5, $P \geq .14$ ). The mean posterior vertical height changes across T8-T10 (or T7-T9) with short fixation were $50 \% \pm 28 \%$ of normal with CS and $52 \% \pm 34 \%$ of normal with PS. With long fixation, mean changes were $40 \% \pm 25 \%$ of normal with CS and $41 \% \pm 17 \%$ with PS. With short fixation and burst fracture, the mean change in vertical height was $301 \% \pm 75 \%$ of normal with CS and $285 \% \pm 228 \%$ with PS. The corresponding change with long fixation and burst fracture was $240 \% \pm 71 \%$ of normal with CS and $241 \% \pm 138 \%$ with PS. Conversely, changes in posterior vertical height during compressive loading were reduced by $33 \%$ with CS and short fixation and by $47 \%$ with PS and short fixation after burst fracture, compared to $47 \%$ with CS and long fixation and $40 \%$ with PS and long fixation.

\section{DISCUSSION}

The standard fusion technique for unstable thoracolumbar fractures is PS-and-rod fixation. ${ }^{3-6}$ The objective of internal fixation is to correct deformity and immobilize the unstable spinal segment to facilitate solid bony fusion. ${ }^{6}$ The traditional PS insertion technique uses a medially angled approach starting at the junction of the transverse process and the lateral wall of the facet. ${ }^{14}$ However, this trajectory requires extensive dissection of paraspinal muscles. Novel screw designs and alternative trajectories such as the CS trajectory can enhance construct stability and improve pullout strength in the lumbar spine. ${ }^{10,14,20}$ In addition to biomechanical advantages, the reduced size of implants and more medial point of insertion minimize muscle retraction, possibly improving recovery time and decreasing postoperative pain. Santoni et al ${ }^{10}$ concluded that a shorter and smaller screw, with a laterally directed cortical bone trajectory, leads to a $30 \%$ increase in resistance to pullout in the lumbar spine, compared with the standard PS trajectory. Their cortical trajectory follows a caudal-cephalad path sagittally, and a laterally directed path in the transverse plane, engaging cortical bone only in the pedicle and anchoring in the superior cortical plate of the vertebral body for tricortical purchase. ${ }^{10,12,14}$ Despite a reported increase in pullout force for CSs compared to PSs, the biomechanics of CS-and-rod fixation and PS-and-rod fixation are reported to be equivalent in terms of construct flexibility in the lumbar spine. ${ }^{3}$ Matsukawa et $\mathrm{al}^{12}$ suggested a biomechanical advantage with CS use compared to traditional PS use in the thoracic spine and reported a $50 \%$ greater insertion torque with CSs. Results from one of our previous studies using thoracic spines, similar to what has been reported in lumbar spines, indicate that improved insertion torque does not necessarily translate to an advantage in terms of improved stability. ${ }^{3}$

Our data showed trends for constructs with PS to be more stable than those with CS during flexionextension and lateral bending, both with short and long fixation, despite greater intact mobility in the PS group (Table 1). However, differences were statistically significant only during lateral bending (Figure 4B). The greater stiffness noted with the PS 
than with the CS construct during lateral bending is likely related to the wider separation between rods that results with the trajectory of PS compared to that of more medially placed CS, which results in rods that are spaced closer together and hence a narrower base of support. As with PS, long-fixation constructs with CS had significantly better stability than short-fixation constructs with CS. This outcome reiterates the inferences in a previous study comparing short- to long-fixation constructs with PS with 4-segment versus 2-segment fixation; it also supports the role of the long-fixation construct in managing patients with severe instability in the thoracolumbar spine. ${ }^{7-9}$ Except for ROM during lateral bending (Figure 4), there were no statistically significant differences in the CS and PS groups in stable or severely unstable conditions, suggesting that CS and rod constructs could be a viable alternative to conventional PS and rod constructs both in the degenerative spine and in cases of severe instability after spinal trauma. For both constructs, compression test results suggested trends for constructs with PS to resist axial compressive loading better than those with CS. However, these differences were not statistically significant.

This study does have several limitations. First, the lack of musculature in cadaveric specimens likely overestimates the degree of destabilization. This excessive instability might lead to underestimation of the stabilizing potential of the instrumentation. Second, smaller-diameter screws were used with CS (4.0-mm diameter) than with PS (4.5-mm diameter) constructs, as is typical clinically. Whether larger-diameter screws accounted for better biomechanical characteristics of the PS-and-rod fixation is unknown, but the screw diameter selected was notably optimum for the condition. Further studies with CS and PS screws of similar diameter may answer these questions. Third, given the number of steps in the study, tissue degradation could have biased results in favor of earlier steps. We attempted to mitigate this factor by varying testing sequence order in different specimens. Fourth, this biomechanical study investigated only the immediate postoperative stability offered by CS and PS constructs under physiological loading. Although some differences were observed between groups, whether these differences would translate to relevant clinical differences is unknown. We assume that greater stability would result in a better environment for fusion. Lastly, the $6.0-\mathrm{Nm}$ maxi- mum load used during the flexibility tests is slightly greater than the European Spine Journal-recommended maximum load of $5.0 \mathrm{Nm}^{21}$ Since the recommended $5.0-\mathrm{Nm}$ load level is meant to be a safe load for the entire thoracic spine, and the levels of interest in the current study are mainly in the lower thoracic spine (T7 to T10), it seems unlikely that $6.0 \mathrm{Nm}$ would have affected study outcome. Similarly, loading during flexibility tests was applied in 1.5-Nm increments (with each load level held 45 seconds) versus the recommended application of 3 continuous load cycles at $0.5^{\circ}$ to $5^{\circ}$ per second. ${ }^{21} \mathrm{An}$ interlaboratory in vitro study involving repeat testing of the same cadaveric spine specimens has shown that this difference in methods has a minimal effect on ROM. ${ }^{22}$

Despite such limitations, this comparison of posterior short-segment and long-segment fixation using a CS trajectory with the standard PS-and-rod fixation technique for the thoracic spine using a 3column spinal injury model produced results that we believe apply to the clinical setting. In simulating immediate postoperative stability conferred by constructs, it showed that posterior instrumentation with either a CS or PS trajectory spanning 2 levels above and 2 levels below a burst fracture in the thoracic spine provides significantly more stability than constructs spanning 1 level above and 1 level below the fracture. These flexibility and compression tests also showed that posterior fixation with a CS trajectory did not provide a statistically significant difference in stability over fixation with standard PS with either a short or long construct for a 3-column spinal injury with a burst fracture at the index level. Thus, on the basis of the immediate postoperative stability alone, these results support CS as a valid alternative to PS for thoracic spine fixation in patients with a 3-column spinal injury.

\section{ACKNOWLEDGMENTS}

The authors thank the staff of Neuroscience Publications at Barrow Neurological Institute for assistance with manuscript preparation.

\section{REFERENCES}

1. Vaccaro AR, Zeiller SC, Hulbert RJ, et al. The thoracolumbar injury severity score: a proposed treatment algorithm. J Spinal Disord Tech. 2005;18(3):209-215.

2. Agus H, Kayali C, Arslantas M. Nonoperative treatment of burst-type thoracolumbar vertebra fractures: clinical and 
radiological results of 29 patients. Eur Spine J. 2005;14(6):536540 .

3. Perez-Orribo L, Kalb S, Reyes PM, Chang SW, Crawford NR. Biomechanics of lumbar cortical screw-rod fixation versus pedicle screw-rod fixation with and without interbody support. Spine (Phila Pa 1976). 2013;38(8):635-641.

4. Cho W, Cho SK, Wu C. The biomechanics of pedicle screwbased instrumentation. J Bone Joint Surg. 2010;92(8):1061-1065.

5. Yue JJ, Sossan A, Selgrath C, et al. The treatment of unstable thoracic spine fractures with transpedicular screw instrumentation: a 3-year consecutive series. Spine (Phila Pa 1976). 2002;27(24):2782-2787.

6. Boos N, Webb JK. Pedicle screw fixation in spinal disorders: a European view. Eur Spine J. 1997;6(1):2-18.

7. McLain RF. The biomechanics of long versus short fixation for thoracolumbar spine fractures. Spine (Phila $\mathrm{Pa}$ 1976). 2006;31(suppl 11):S70-S79; discussion S104.

8. Sodhi HBS, Savardekar AR, Chauhan RB, Patra DP, Singla N, Salunke P. Factors predicting long-term outcome after short-segment posterior fixation for traumatic thoracolumbar fractures. Surg Neurol Int. 2017;8:233.

9. Lazaro BC, Deniz FE, Brasiliense LB, et al. Biomechanics of thoracic short versus long fixation after 3-column injury. $J$ Neurosurg Spine. 2011;14(2):226-234.

10. Santoni BG, Hynes RA, McGilvray KC, et al. Cortical bone trajectory for lumbar pedicle screws. Spine J. 2009;9(5):366373.

11. Snyder LA, Martinez-Del-Campo E, Neal MT, et al. Lumbar spinal fixation with cortical bone trajectory pedicle screws in 79 patients with degenerative disease: perioperative outcomes and complications. World Neurosurg. 2016;88:205213.

12. Matsukawa K, Yato Y, Hynes RA, et al. Cortical bone trajectory for thoracic pedicle screws: a technical note. Clin Spine Surg. 2017;30(5):E497-E504.

13. Chin KR, Pencle FJR, Coombs AV, et al. Clinical outcomes with midline cortical bone trajectory pedicle screws versus traditional pedicle screws in moving lumbar fusions from hospitals to outpatient surgery centers. Clin Spine Surg. 2017;30(6):E791-E797.

14. Mullin JP, Perlmutter B, Schmidt E, Benzel E, Steinmetz MP. Radiographic feasibility study of cortical bone trajectory and traditional pedicle screw dual trajectories. J Neurosurg Spine. 2016;25(6):727-732.

15. Crawford NR, Brantley AG, Dickman CA, Koeneman EJ. An apparatus for applying pure nonconstraining moments to spine segments in vitro. Spine (Phila Pa 1976). 1995;20(19):2097-2100.

16. Panjabi MM. Biomechanical evaluation of spinal fixation devices: I. A conceptual framework. Spine (Phila Pa 1976). 1988;13(10):1129-1134.

17. Crawford NR, Dickman CA. Construction of local vertebral coordinate systems using a digitizing probe: technical note. Spine (Phila Pa 1976). 1997;22(5):559-563.

18. Crawford NR, Yamaguchi GT, Dickman CA. A new technique for determining 3-D joint angles: the tilt/twist method. Clin Biomech. 1999;14(3):153-165.

19. Crawford NR, Peles JD, Dickman CA. The spinal lax zone and neutral zone: measurement techniques and parameter comparisons. J Spinal Disord. 1998;11(5):416-429.

20. Inceoglu S, Montgomery WH, Jr, St Clair S, McLain RF. Pedicle screw insertion angle and pullout strength: comparison of 2 proposed strategies. J Neurosurg Spine. 2011;14(5):670-676.

21. Wilke HJ, Wenger K, Claes L. Testing criteria for spinal implants: recommendations for the standardization of in vitro stability testing of spinal implants. Eur Spine J. 1998;7(2):148154.

22. Wheeler DJ, Freeman AL, Ellingson AM, et al. Interlaboratory variability in in vitro spinal segment flexibility testing. J Biomech. 2011;44(13):2383-2387.

Disclosures and COl: The authors have nothing to disclose. This study was funded internally. Implants and instrumentation were donated by Medtronic, plc.

Corresponding Author: Neil R. Crawford, $\mathrm{PhD}$, c/o Neuroscience Publications; Barrow Neurological Institute, St Joseph's Hospital and Medical Center, 350 W Thomas Road; Phoenix, AZ 85013. Phone: (602) 406-3593; Fax: (602) 406-4104; Email: neuropub@barrowneuro.org

Published 30 June 2019

This manuscript is generously published free of charge by ISASS, the International Society for the Advancement of Spine Surgery. Copyright (c) 2019 ISASS. To see more or order reprints or permissions, see http://ijssurgery.com. 